\title{
MORPHOMETRIC STUDIES OF RADIOLARIAN EVOLUTION
}

LAZARUS*, Dave, Geologisches Institut, ETH-Zentrum, Zürich, Switzerland, CH-8092.

Radiolarians are the most diverse of the 4 major fossil forming plankton groups, with several hundred living species, and an equally diverse range of biologic specializations for planktonic existence. Studies of radiolarian evolution are mostly from the Cenozoic, where the opaline silica skeletons of radiolarians are well preserved in most areas of the world's oceans. Cenozoic radiolarian skeletons are mostly too small $(50-150 \mu \mathrm{m})$ and delicate to easily manipulate individually. Washed sieved skeletons are strewn at random orientations onto glass microslides and viewed in transmitted light. At the required magnifications, most skeletons cannot be viewed in their entirety within a single focus-plane. Many (perhaps the majority) of taxonomic characters in radiolarians are not visible in the skeleton outline, but are internal. These characteristics make normal image analysis techniques more difficult to employ. Computer assisted manual digitizers (one version working in 3 dimensions) have sometimes been used to measure skeletons. Distance, angle, area, and shape outline measurements have all been used to quantify radiolarian morphology.

Radiolarian skeletal growth is determinate, and relatively little ontogenetic or within-population allometric variation is seen. Geographic variation in radiolarian species morphology is common, but little studied. Perhaps due to the effort involved, most ordinary taxonomic work does not employ morphometric data: its use has largely been in species-level evolutionary studies. Phyletic trends in size, shape, and in other characters have long been used to delineate stratigraphically useful chronospecies, and some of these anagenetic series have been morphometrically measured. Cladogenesis has also been morphometrically quantified in a few cases. Most studies of both cladogenesis and anagenetic evolution have documented gradual rates of morphologic change.

Integrated studies of paleogeographic variation and temporal change are still needed, as is better information on modern radiolarian biology. Much more efficient measurement tools are also needed, to handle the very large number of specimens required by integrated studies. 